Originalien

Ophthalmologe 2021 · 118:670-674 https://doi.org/10.1007/s00347-020-01248-6 Eingegangen: 3 . Juni 2020

Überarbeitet: 6. August 2020

Angenommen: 21. September 2020

Online publiziert: 10 . Oktober 2020

(c) Der/die Autor(en) 2020, korrigierte

Publikation 2021

N. Kaupke $($ D $\cdot$ M. S. Spitzer $\cdot$ R. Kromer

Klinik und Poliklinik für Augenheilkunde, Universitätsklinikum Hamburg-Eppendorf (UKE), Hamburg, Deutschland

\title{
Amotio-retinae-Versorgung während der Corona-Pandemie
}

\section{Suchten Patienten mit Netzhautablösung während der Corona-Pandemie später den Augenarzt auf? Ergebnisse aus einer deutschen Universitätsaugenklinik}

\begin{abstract}
Eine Netzhautablösung stellt einen ophthalmologischen Notfall dar. Eine Verzögerung der Diagnose oder Therapie kann die Visusprognose reduzieren. Aus aktuellen Presseberichten geht hervor, dass Notfallpatienten beispielsweise mit Myokardinfarkt oder Apoplex aus Angst vor einer Ansteckung mit dem Coronavirus erst deutlich verspätet einen Arzt aufsuchen und somit die Diagnose und Therapie verschleppen. Die vorliegende Arbeit untersucht, ob die Corona-Pandemie Auswirkung auf die Versorgung und den präoperativen Visus von Patienten mit Netzhautablösung hatte.
\end{abstract}

Eine Amotio retinae ist ein ophthalmologischer Notfall und führt unbehandelt zu einem progredienten Visusverlust [7, $10,16,21,24]$. Die häufigste Form ist dabei eine rhegmatogene Amotio retinae. Ziel der primär operativen Therapie ist das Wiederanlegen der Netzhaut [24]. Ein anatomischer Erfolg wird dabei in $85-90 \%$ der Operationen erreicht $[9,16$, 17, 21]. Der funktionelle Erfolg ist v. a. abhängig von einer Mitbeteiligung der Makula [6, 13]. Das Risiko einer Makulabeteiligung steigt dabei mit zunehmender Dauer der Netzhautablösung signifikant an $[1,4,9]$.

In der Regel wird eine Netzhautablösung nicht im Rahmen einer augenärzt- lichen Routineuntersuchung, sondern durch den Patienten selbst bemerkt [7, 11]. Zwar führen auch verspätete ärztliche Diagnosen und organisatorische Abläufe zu einer verzögerten Therapie, die anteilig größte Verzögerung entsteht jedoch durch ein verspätetes Aufsuchen des Arztes durch den Patienten [11]. Somit kommt dem Patienten eine große Verantwortung für eine zeitnahe Behandlung zu.

Die jährliche Inzidenz einer Amotio retinae beträgt in Europa etwa 1 Fall pro 10.000 Einwohner [15, 24]. Damit betrifft diese Erkrankung jährlich etwa $8300 \mathrm{~Pa}-$ tienten in Deutschland und ist einer der häufigsten ophthalmologischen Notfälle mit akuter Gefahr für die Sehkraft [7, 25]. Das Risiko einer Netzhautablösung steigt mit zunehmenden Alter, insbesondere ab dem 50. Lebensjahr [18, 19]. Damit betrifft dieses Erkrankungsbild v. a. das Patientenkollektiv, das auch ein deutlich erhöhtes Risiko für einen schweren Verlauf einer COVID-19-Infektion hat $[14,26]$.

Aus aktuellen Presseberichten geht hervor, dass Notfallpatienten z. B. mit Myokardinfarkt oder Apoplex aus Angst vor einer Ansteckung mit dem Coronavirus SARS-CoV-2 erst deutlich verspätet einen Arzt aufsuchen und somit Diagnose und Therapie verschleppen [8, 12]. Diese Arbeit soll mittels retrospektiver Analyse von operativ versorgten Netzhautablösungen einer deutschen
Universitätsklinik untersuchen, ob sich Patienten mit Netzhautablösung während der Corona-Pandemie verspätet beim Augenarzt vorstellen und ob dies eine Auswirkung auf die Schwere der Amotio retinae hat.

\section{Material und Methoden}

Für diese Studie wurden alle Patienten mit einer im Zeitraum 15.03.-05.05.2020 in der Augenklinik des Universitätsklinikum Hamburg-Eppendorf operativ versorgten rhegmatogenen Amotio retinae eingeschlossen $(n=60$, mittleres Alter: 57,5 \pm 14,6 Jahre). Als Studienbeginn wurde das Datum des Inkrafttretens einer weitreichenden Allgemeinverfügung gewählt, die zum Schutz vor der Corona-Pandemie das öffentliche Leben in Hamburg deutlich einschränkte [3]. Der Studienzeitraum endete mit der Gültigkeit der Allgemeinverfügung und fiel mit der schrittweisen Lockerung der bundesweiten Ausgangsbeschränkungen zusammen [2]. Als Vergleichsgruppe wurden Patienten aus dem entsprechenden Vorjahreszeitraum eingeschlossen ( $n=48$, mittleres Alter: 62,5 \pm 12,3 Jahre). Von der Studie ausgeschlossen wurden Fälle mit erneuter Netzhautablösung am gleichen Auge sowie während eines stationären Aufenthaltes aufgetretenen Netzhautablösungen. Die Datenerhebung erfolgte retrospektiv und anonymisiert. Neben Alter, Geschlecht und 


\begin{tabular}{|c|c|c|c|}
\hline & $\begin{array}{l}\text { Corona-Pandemie } 2020 \\
n=60\end{array}$ & $\begin{array}{l}\text { Kontrollzeitraum } 2019 \\
n=48\end{array}$ & $p$-Wert \\
\hline Geschlecht & $30 \%$ weiblich & $33 \%$ weiblich & $0,2020^{\mathrm{a}}$ \\
\hline Alter bei Operation (Jahre) & $57,5 \pm 14,6$ & $62,5 \pm 12,3$ & $0,8712^{b}$ \\
\hline Betroffenes Auge & $45 \%$ rechtes Auge & $44 \%$ rechtes Auge & $0,8966^{b}$ \\
\hline Symptombeginn (Tage bis zur Operation) & $7,0 \pm 8,4$ & $7,6 \pm 11,8$ & $0,6040^{\mathrm{a}}$ \\
\hline Vorhergehender Augenarztbesuch & $54 \%$ ja (insgesamt $n=46$ ) & $49 \%$ ja (insgesamt $n=43$ ) & $0,7584^{b}$ \\
\hline Visus präoperativ (LogMAR) & $1,1 \pm 0,8$ & $1,0 \pm 0,9$ & $0,4155^{\mathrm{a}}$ \\
\hline Makulabeteiligung & $57 \%$ ja & $58 \%$ ja & $0,8618^{b}$ \\
\hline Betroffene Uhrzeiten (h) & $6,4 \pm 2,3$ & $6,4 \pm 2,3$ & $0,9019^{\mathrm{a}}$ \\
\hline PVR & $22 \%$ ja & $19 \%$ ja & $0,8938^{\mathrm{b}}$ \\
\hline Operationsverfahren & $\begin{array}{l}48 \% \text { ppV mit Gas } \\
47 \% \text { ppV mit Öl } \\
5 \% \text { Plombe }\end{array}$ & $\begin{array}{l}67 \% \text { ppV mit Gas } \\
31 \% \text { ppV mit Öl } \\
2 \% \text { Plombe }\end{array}$ & $0,1502^{c}$ \\
\hline Operationsdauer (min) & $43 \pm 15,4$ & $39 \pm 10,5$ & $0,2117^{\mathrm{a}}$ \\
\hline \multicolumn{4}{|c|}{ 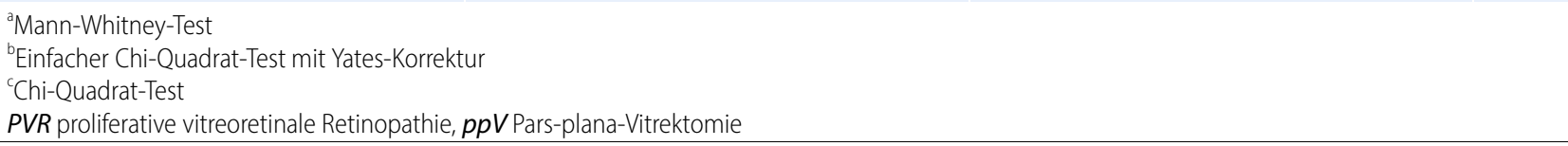 } \\
\hline
\end{tabular}

betroffenem Auge wurde der Visus als bester korrigierter Visus bei Vorstellung im Krankenhaus erfasst. Eine Beurteilung der Makulabeteiligung erfolgte mittels optischer Kohärenztomographie (OCT). War eine OCT-Untersuchung nicht möglich, wurde der Makulastatus ebenso wie das Ausmaß der Amotio retinae anhand des fundoskopischen Befunds und ggf. weiterer apparativer Diagnostik (z. B. Weitwinkelfundusfotografie, Echographie) erhoben. Aus den Operationsberichten wurden das Vorhandensein von proliferativer Vitreoretinopathie, das Operationsverfahren (Pars-plana-Vitrektomie mit Silikonöl oder Gas, Plombenoperation mit Kryoretinopexie) sowie die Operationsdauer erhoben.

In der klinischen Routine wurden ohne standardisierten Fragebogen weiterhin erfragt und ausgewertet:

1. Anamnestische Symptomdauer in Tagen bis zur Vorstellung in der Klinik.

2. Erfolgte bereits ein ambulanter Augenarztbesuch aufgrund der Symptome?

3. Erfolgte die Vorstellung in der Augenklinik aufgrund der CoronaPandemie verzögert? (Zum Beispiel: Konkrete Verzögerung aufgrund geänderter Öffnungszeiten des Augenarztes? Verzögerung aus Angst vor Ansteckung? Bestehen allgemeine Gesundheitssorgen aufgrund der Pandemie?)

Die statistische Auswertung erfolgte mittels SPSS 25 (IBM, New York, USA). Signifikante Gruppenunterschiede wurden mittels Hypothesentests identifiziert. Die Normalverteilung von ordinalskalierten Daten wurde mittels D’Agostino \& Pearson-Test geprüft. Falls keine Normalverteilung vorlag, wurde der Mann-Whitney-Test verwendet, sonst der ungepaarte t-Test. Kategoriale Variablen wurden mittels des einfachen Chi-Quadrat-Tests mit Yates-Korrekturbzw. beimehr als 2 Variablen mittels des Chi-Quadrat-Tests verglichen. Es wurde ein Signifikanzniveau von $p=0,05$ gewählt. Um eine statistische Auswertung zu ermöglichen, erfolgte eine Transformation der Visuswerte in logMAR [22]. Dabei entspricht beispielsweise der Visus von 1,0 im Dezimalsystem einem LogMAR von 0 und ein Dezimalvisus von 0,1 einem LogMAR von 1.

\section{Ergebnisse}

Im 7-wöchigen Studienzeitraum (15.03.-05.05.2020) wurden während der Corona-Pandemie im Universitätsklinikum Hamburg-Eppendorf 60 Patienten mit rhegmatogener Amotio retinae operativ versorgt. Im gleich langen
Vorjahreszeitraum (2019) waren es 48. Im Vergleich zum Vorjahreszeitraum zeigten sich keine signifikanten Unterschiede der Patientencharakteristika Geschlecht, Alter, betroffenes Auges, Symptomdauer, vorheriger Arztbesuch, präoperativer Visus, Makulabeteiligung, Amotioausdehnung, Vorhandensein von proliferativer vitreoretinaler Retinopathie (PVR), Operationsverfahren und Operationsdauer (• Tab. 1).

Während der Corona-Pandemie gaben $29 \%$ der diesbezüglich befragten Patienten $(n=35)$ an, sie hätten aufgrund des Corona-Virus grundsätzlich Sorge um ihre Gesundheit. Der einzige Patient, der eine verzögerte ärztliche Vorstellung aus Sorge vor einer Ansteckung mit dem COVID-19-Virus angab, bemerkte erst seit 1 Tag Symptome. Bei Vorstellung in der Augenklinik gaben Patienten ohne Gesundheitssorgen aufgrund der Corona-Pandemie $(n=25)$ eine Symptomdauer von 6,4 $\pm 6,9$ Tagen und einen präoperativen Visus (LogMAR) von $1,1 \pm 0,8$ an. Patienten mit pandemiebedingten Gesundheitssorgen $(n=10)$ zeigten eine Symptomdauer von $4,1 \pm 3,3$ Tagen und einen präoperativen Visus (LogMAR) von 1,0 $\pm 0,9$. Die Unterschiede der Symptomdauer $(p=0,5)$ und des präoperativen Visus $(p=0,44)$ zeigten sich statistisch nicht signifikant (Mann-Whitney-U-Test). 
Ophthalmologe 2021 · 118:670-674 https://doi.org/10.1007/s00347-020-01248-6

(c) Der/die Autor(en) 2020

\section{N. Kaupke $\cdot$ M. S. Spitzer $\cdot$ R. Kromer}

\section{Amotio-retinae-Versorgung während der Corona-Pandemie. Suchten Patienten mit Netzhautablösung während der Corona-Pandemie später den Augenarzt auf? Ergebnisse aus einer deutschen Universitätsaugenklinik}

\section{Zusammenfassung}

Hintergrund. Eine Amotio retinae ist ein ophthalmologischer Notfall. Verzögerung der Diagnose oder Therapie kann die Visusprognose reduzieren. Aus Presseberichten geht hervor, dass Notfallpatienten aus Angst vor einer Ansteckung mit dem Coronavirus SARS-CoV-2 erst deutlich verspätet einen Arzt aufsuchen und die Diagnose und Therapie verschleppen. Diese Arbeit untersucht, ob die Corona-Pandemie Auswirkungen auf die Versorgung von Patienten mit Netzhautablösung hatte.

Methodik. Für diese Studie wurden $60 \mathrm{~Pa}$ tienten mit im Zeitraum 15.03.-05.05.2020 in der Augenklinik des Universitätsklinikum Hamburg-Eppendorf operativ versorgter rhegmatogener Netzhautablösung retrospektiv eingeschlossen. Als Vergleichszeitraum wurde der entsprechende Vorjahreszeitraum gewählt. Signifikante Unterschiede zwischen den Vergleichsgruppen wurden mittels Hypothesentests untersucht.

Ergebnisse. Im Vergleich zum Vorjahreszeitraum zeigten sich während der CoronaPandemie keine signifikanten Unterschiede von Geschlecht, Alter, Symptomdauer, vorherigem Arztbesuch, präoperativem Visus, Makulabeteiligung, Amotioausdehnung, Vorhandensein von proliferativer vitreoretinaler Retinopathie (PVR), Operationsverfahren oder Operationsdauer. Während der CoronaPandemie gaben $29 \%$ der befragten Patienten mit Amotio retinae generelle Sorgen um ihre Gesundheit aufgrund des Coronavirus SARSCoV-2 an.

Diskussion. Während der Corona-Pandemie zeigte sich eine unveränderte Versorgung von Patienten mit Amotio retinae. Im Gegensatz zu anderen medizinischen Notfällen zeigte sich keine reduzierte Fallzahl oder erhöhte Morbidität im Vergleich zum Vorjahreszeitraum.

Schlüsselwörter

Netzhautablösung · COVID-19 · SARS-CoV-2 . Deutschland · Ophthalmologischer Notfall

\section{Treatment of retinal detachment during the COVID-19 pandemic. Did patients with retinal detachment seek treatment later during the COVID-19 pandemic? Results from a German university eye hospital}

\begin{abstract}
Background. Retinal detachment is an ophthalmological emergency. Delayed diagnostics and treatment increase the risk of permanent loss of vision. Current media reports have suggested that patients with medical emergencies delay seeking treatment out of fear of being infected with the corona virus SARS-CoV-2. This study analyzed data from a German university hospital to determine if the coronavirus pandemic had an impact on treatment and visual outcomes of patients with retinal detachment.

Methods. In this study 60 patients treated for rhegmatogenous retinal detachment in the eye hospital of the University Hospital
\end{abstract}

Hamburg-Eppendorf between 15 March and 5 May 2020 were retrospectively analyzed. Patients from the corresponding period of the previous year acted as a control group. Significant differences between the groups were investigated by hypothesis testing. Results. When compared to the period in the previous year there were no significant differences for sex, age, eye, length of symptoms, previous visit to doctor, visual acuity, macula status, degree of retinal detachment, proliferative vitreoretinopathy and type or length of procedure during the coronavirus pandemic. Of the patients with retinal detachment $29 \%$ had general health concerns due to the coronavirus pandemic. Conclusion. Medical treatment for retinal detachment was not influenced by the coronavirus pandemic. In contrast to other medical emergencies the morbidity of retinal detachment did not increase and admitted cases did not decrease during the pandemic when compared to the same period in the previous year.

\section{Keywords}

Retinal detachment · SARS-CoV-2 - COVID-19. Ophthalmological emergency · Germany

\section{Diskussion}

Wir konnten zeigen, dass Patienten mit rhegmatogener Amotio retinae während der Corona-Pandemie im Vergleich zum Vorjahreszeitraum weder ein signifikant längeres Symptomintervall bis zur Vorstellung in der Augenklinik noch einen schlechteren präoperativen Visus aufwiesen. Während der Corona-Pandemie zeigten sich für Patienten mit bzw. ohne Gesundheitssorgen aufgrund der Corona-Pandemie keine signifikanten
Unterschiede von Symptomdauer und präoperativem Visus.

Die durchschnittliche Symptomdauer bis zur Vorstellung in der Klink war in der vorliegenden Untersuchung geringer als in der Literatur beschrieben [11, 20, 21]. Ein Vergleich mit Gesundheitssystemen, in denen der Hausarzt als Gatekeeper vor einem Facharztbesuch fungiert, ist jedoch nur bedingt aussagekräftig.

Mit Ausnahme einer größeren Patientenanzahl zeigten sich keine statistisch relevanten Unterschiede zum Vorjahres- zeitraum. Eine tatsächliche Erhöhung der Fallzahlen kann in dieser Arbeit nicht ausgeschlossen werden. Das erhöhte Patientenaufkommen in der Studienklinik ist jedoch wahrscheinlich auf reduzierte Kapazitäten der umliegenden Kliniken im Rahmen von Coronavirus-Beschränkungen zurückzuführen.

Den Autoren ist keine weitere Untersuchung der Auswirkungen der CoronaPandemie auf ophthalmologische Notfälle bekannt. Untersuchungen anderer medizinischer Notfälle aus den USA 
und Italien zeigten während der CoronaPandemie jedoch eine annähernde Halbierung der Hospitalisierungsraten für Myokardinfarkte bei erhöhter Mortalität und Morbidität der Fälle [5, 23]. Warum sich das Patientenverhalten zwischen verschiedenen medizinischen Notfällen unterscheidet, lässt sich nur mutmaßen. Zum einen waren die USA und Italien von der Corona-Pandemie deutlich schwerer betroffen, sodass medizinische Kapazitäten ggf. eingeschränkter und die Sorge vor einer Ansteckung als Hindernis für ein Aufsuchen des Krankenhauses möglicherweise größer waren als in Deutschland. Zum anderen zeigten in unserer Arbeit Patienten trotz genereller Gesundheitssorgen aufgrund der Corona-Pandemie kein anderes Verhalten als Patienten ohne diese Sorgen. Möglicherweise überwogen die Sorgen vor Sehverlust gegenüber einer potenziellen Ansteckung mit dem Coronavirus, während die unterschiedlich stark ausgeprägten Beschwerden eines Myokardinfarktes nicht im gleichen Maße als ernst wahrgenommen und zunächst abgewartet wurden.

Zusammenfassend zeigten sich in dieser Arbeit im Vergleich zum Vorjahreszeitraum während der Corona-Pandemie erfreulicherweise keine signifikanten Unterschiede in der Schwere der Fälle oder der Versorgung von Patienten mit Amotio retinae. Unsere Ergebnisse geben damit Anhalt dafür, dass die Versorgung von ophthalmologischen Notfallpatienten während der Corona-Pandemie auf einem hohen Niveau aufrechterhalten werden konnte.

\section{Limitationen}

Aufgrund der besonderen Situation während der Corona-Pandemie erfolgte eine retrospektive Untersuchung klinischer Routinedaten über einen zeitlich begrenzten Zeitraum. Es wurde eine begrenzte Studienpopulation eines variablen Krankheitsbildes untersucht. Eine Interpretation der Auswertung der nicht standardisierten Aufzeichnungen zur Anamnese sollte zurückhaltend erfolgen. Über die Ursache der im Vergleich zum Vorjahreszeitraum erhöhten Fallzahl lässt sich nur mutmaßen. Ein ver- ändertes Verhalten von Zuweisern und umliegenden Kliniken ist eine mögliche Ursache und sollte in weiteren Arbeiten untersucht werden. Eine Untersuchung des postoperativen Verlaufs oder weiterer ophthalmologischer Notfälle erfolgte nicht.

\section{Ausblick}

Die Ergebnisse dieser Arbeit geben Anhalt zu vermuten, dass sich die Versorgung von Patienten mit dem ophthalmologischen Notfall einer Netzhautablösung während der Corona-Pandemie nicht signifikant verschlechterte. Ob sich dies auch in einer Analyse des langfristigen funktionellen und anatomischen Erfolgs bestätigt, bleibt abzuwarten. Zur Bestätigung der vorliegenden Ergebnisse sollte eine multizentrische Arbeit mit größerer Studienpopulation auch das Notfallmanagement unterschiedlicher Kliniken beleuchten.

\section{Fazit für die Praxis}

\section{- Patienten mit Symptomen einer Netzhautablösung zögerten trotz der Corona-Pandemie nicht, den Augen- arzt aufzusuchen, und die Schwere der behandelten Netzhautablösun- gen unterschied sich nicht signifikant zum Vorjahreszeitraum. \\ - Während der Corona-Pandemie konnte die Versorgung von Patienten mit Amotio retinae auf einem hohen Niveau aufrechterhalten werden.}

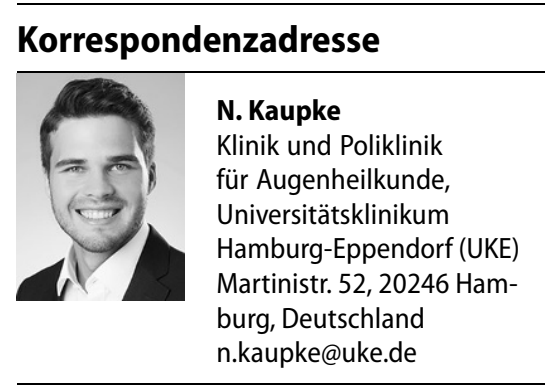

Funding. Open Access funding enabled and organized by Projekt DEAL

\section{Einhaltung ethischer Richtlinien}

Interessenkonflikt. N. Kaupke, M.S. Spitzer und R. Kromer geben an, dass kein Interessenkonflikt besteht.

Es erfolgte eine anonymisierte retrospektive Analyse klinischer Daten. Im Einklang mit nationalem Recht sowie der Deklaration von Helsinki von 1975 ist keine Beurteilung der Ethikkommission erforderlich.

Open Access. Dieser Artikel wird unter der Creative Commons Namensnennung 4.0 International Lizenz veröffentlicht, welche die Nutzung, Vervielfältigung, Bearbeitung, Verbreitung und Wiedergabe in jeglichem Medium und Format erlaubt, sofern Sie den/die ursprünglichen Autor(en) und die Quelle ordnungsgemäß nennen, einen Link zur Creative Commons Lizenz beifügen und angeben, ob Änderungen vorgenommen wurden.

Die in diesem Artikel enthaltenen Bilder und sonstiges Drittmaterial unterliegen ebenfalls der genannten Creative Commons Lizenz, sofern sich aus der Abbildungslegende nichts anderes ergibt. Sofern das betreffende Material nicht unter der genannten Creative Commons Lizenz steht und die betreffende Handlung nicht nach gesetzlichen Vorschriften erlaubt ist, ist für die oben aufgeführten Weiterverwendungen des Materials die Einwilligung des jeweiligen Rechteinhabers einzuholen.

Weitere Details zur Lizenz entnehmen Sie bitte der Lizenzinformation auf http://creativecommons.org/ licenses/by/4.0/deed.de.

\section{Literatur}

1. Angermann $\mathrm{R}$, Bechrakis NE, Rauchegger $T$ et al (2020) Effect of timing on visual outcomes in foveainvolving retinal detachments verified by SD-OCT. JOphthalmol 2020:2307935

2. Behörde Für Gesundheit Und Verbraucherschutz (2020) Verordnung zur Eindämmung der Ausbreitung des Coronavirus SARS-CoV-2 in der Freien und Hansestadt Hamburg (Hamburgische SARSCoV-2-Eindämmungsverordnung - HmbSARSCoV-2-EindämmungsVO) Vom 2. April 2020 (gültig ab 6. Mai 2020). Behörde Für Gesundheit Und Verbraucherschutz, Hamburg

3. Behörde Für Gesundheit Und Verbraucherschutz Hamburg (2020) Allgemeinverfügung zur Eindämmung des Coronavirus in Hamburg 15. März 2020. Behörde Für Gesundheit Und Verbraucherschutz, Hamburg

4. Callizo J, Pfeiffer S, Lahme E et al (2017) Risk of progression in macula-on rhegmatogenous retinal detachment. Graefes Arch Clin Exp Ophthalmol 255:1559-1564

5. De Rosa S, Spaccarotella C, Basso C et al (2020) Reduction of hospitalizations for myocardial infarction in Italy in the COVID-19 era. Eur Heart J 41(22):2083-2088. https://doi.org/10. 1093/eurheartj/ehaa409

6. Doyle E, Herbert EN, Bunce C et al (2007) How effective is macula-off retinal detachment surgery. Might good outcome be predicted? Eye (Lond) 21:534-540

7. Feltgen N, WalterP(2014)Rhegmatogenous retinal detachment-an ophthalmologic emergency. Dtsch Arztebl Int 111:12-21 (quiz 22) 
8. Fricke A (2020) Selbst Patienten mit Schlaganfall bleiben zu Hause. Ärzte Z. https://www. aerztezeitung.de/Politik/Selbst-Patienten-mitSchlaganfall-bleiben-zu-Hause-408354.html

9. Gerding H, Hersener A (2013) Anatomical and functional results of primary pars plana vitrectomy in rhegmatogenous retinal detachment. Klin Monatsbl Augenheilkd 230:409-412

10. Gishti O, Van Den Nieuwenhof R, Verhoekx J et al (2019) Symptoms related to posterior vitreous detachment and the risk of developing retinal tears: a systematic review. Acta Ophthalmol 97:347-352

11. Goezinne F, La Heij EC, Berendschot TT et al (2009) Patient ignorance is the main reason for treatment delay in primary rhegmatogenous retinal detachment in The Netherlands. Eye (Lond) 23:1393-1399

12. Hommel T (2020) Verschleppte Krankheiten oft gefährlicher als Corona. Springer, Berlin Heidelberg

13. Lecleire-Collet A, Muraine M, Menard JF et al (2005) Predictive visual outcome after macula-off retinal detachment surgery using optical coherence tomography. Retina 25:44-53

14. Mehra MR, Desai SS, Kuy S et al (2020) Cardiovascular disease, drug therapy, and mortality in Covid-19. N Engl J Med 382:e102. https://doi.org/ 10.1056/NEJMoa2007621

15. Mitry D, Charteris DG, Fleck BW et al (2010) The epidemiology of rhegmatogenous retinal detachment: geographical variation and clinical associations. Br J Ophthalmol 94:678-684

16. Mitry D, Fleck BW, Wright AF et al (2010) Pathogenesis of rhegmatogenous retinal detachment: predisposing anatomy and cell biology. Retina 30:1561-1572

17. Murtagh PJ, Stephenson KA, Rhatigan M et al (2020) Rhegmatogenous retinal detachments: primary reattachment rates and visual outcomes over a 4-year period. Ir J Med Sci 189:355-363

18. Nielsen BR, Alberti M, Bjerrum SS et al (2020) The incidence of rhegmatogenous retinal detachment is increasing. Acta Ophthalmol 98(6):603-606. https://doi.org/10.1111/aos.14380

19. Potic J, Bergin C, Giacuzzo C et al (2018) Primary rhegmatogenous retinal detachment: risk factors for macular involvement. Graefes Arch Clin Exp Ophthalmol 256:489-494

20. Quinn SM, Qureshi F, Charles SJ (2004) Assessment of delays in presentation of patients with retinal detachment to a tertiary referral centre. Ophthalmic Physiol Opt 24:100-105

21. Schmidt I, Plange N, Rossler G et al (2019) Long-term clinical results of vitrectomy and scleral buckling in treatment of rhegmatogenous retinal detachment. ScientificWorldJournal 2019:5416806

22. Schulze-Bonsel K, Feltgen N, Burau H et al (2006) Visual acuities "hand motion" and "counting fingers" can be quantified with the Freiburg visual acuity test. Investig Ophthalmol Vis Sci 47:1236-1240

23. Solomon MD, Mcnulty EJ, Rana JS et al (2020) The Covid-19 pandemic and the incidence of acute myocardial infarction. N Engl J Med 383:88-9. https://doi.org/10.1056/NEJMc2015630

24. Steel D, Fraser S (2008) Retinal detachment. BMJ Clin Evid 2008:0710. PMID: 19450333; PMCID: PMC2907822

25. Van De Put MAJ, Hooymans JMM, Los LI et al (2013) The incidence of rhegmatogenous retinal detachment in The Netherlands. Ophthalmology 120:616-622
26. Weiss P, Murdoch DR (2020) Clinical course and mortality risk of severe COVID-19. Lancet 395:1014-1015

\section{SpringerMedizin.de Lesen Sie Ihre Fachzeitschrift auch als ePaper!}

Als Abonnent können Sie Ihre Zeitschrift in verschiedenen Formaten lesen. Wählen Sie je nach Vorliebe und Situation aus, ob Sie die Zeitschrift als Print-Ausgabe, in Form von einzelnen Beiträgen auf springermedizin.de oder aber als komplette, elektronische ePaper-Ausgabe lesen möchten.

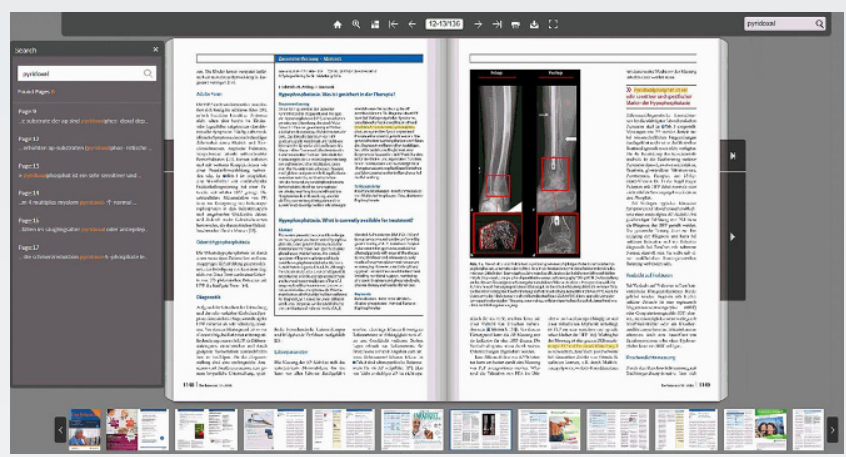

Die ePaper sind die identische Form der gedruckten Ausgaben. Sie sind nutzbar auf verschiedenen Endgeräten wie PC, Tablet oder Smartphone

Das sind die Vorteile des ePapers:

$>$ Das verlinkte Inhaltsverzeichnis führt Sie direkt zum gewünschten Beitrag.

$>$ Eine Suchfunktion ermöglicht das Auffinden von Schlagworten innerhalb der Zeitschrift.

> Jede Ausgabe kann als PDF heruntergeladen und damit auch offline gelesen werden bzw. auch gespeichert oder ausgedruckt werden.

Als Abonnent haben Sie Zugang zu allen ePaper-Ausgaben ab 2016.

Sie finden die ePaper auf SpringerMedizin.de bei der jeweiligen Ausgabe Ihrer Fachzeitschrift. Klicken Sie auf den Button „Ausgabe als ePaper lesen“. 